\title{
Reanalysis of the Radii of the Benchmark Eclipsing Binary V578 Mon
}

\author{
E.V. Garcia ${ }^{1,2}$, Keivan G. Stassun ${ }^{1}$, Guillermo Torres ${ }^{3}$
}

\begin{abstract}
V578 Mon is an eclipsing binary system in which both stars have masses above $10 \mathrm{M}_{\odot}$ determined with an accuracy better than $3 \%$. It is one of only five such massive eclipsing binaries known that also possess eccentric orbits and measured apsidal motions, thus making it an important benchmark for theoretical stellar evolution models. However, recently reported determinations of the radii of V578 Mon differ significantly from previously reported values. We reanalyze the published data for V578 Mon and trace the discrepancy to the use of an incorrect formulation for the stellar potentials in the most recent analysis. Here we report corrected radii for this important benchmark eclipsing binary.
\end{abstract}

\section{Introduction}

In a recent major review of eclipsing binary (EB) stars, Torres et al. (2010) compiled a sample of 95 "benchmark grade" EBs having masses and radii measured with an accuracy of better than $\pm 3 \%$. This sample of EBs is of fundamental importance to a broad range of applications in stellar astrophysics. For example, using this sample, Torres et al. (2010) derive empirical relations that permit the determination of fundamental properties, such as mass and radius, for any star from its directly observable properties, such as effective temperature, surface gravity and metallicity. This sample of EBs is moreover important for testing the basic predictions of theoretical stellar evolution models, which are in turn used across many areas of research, including star formation, stellar evolution, and exoplanets (because the inferred properties of the planets generally depend on the assumed properties of the host stars).

One of the 95 EBs included in the Torres et al. (2010) compilation is V578 Mon. This EB is remarkable by virtue of being one of only nine EBs in the compilation where both stars have masses greater than 10 $\mathrm{M}_{\odot}$, and of these, is one of only five to have an eccentric orbit and therefore a measurable apsidal motion (Hensberge et al. 2000; Pavlovski \& Hensberge 2005). Indeed, the apsidal motion of V578 Mon was only recently measured (Garcia et al. 2011).

We have identified an error in the calculation of the stellar radii reported in the Torres et al. (2010) compilation. In this brief report, we trace the source of this error to the use of a formulation for the stellar potentials that does not correctly include the system's orbital eccentricity. We then provide updated radii calculated from the correct formulation for the potentials.

\section{Calculation of Stellar Radii for V578 Mon}

Torres et al. (2010) reported the radii of V578 Mon to be $R_{p}=5.149 \pm 0.091$ and $R_{s}=4.21 \pm 0.10$,

\footnotetext{
${ }^{1}$ Department of Physics \& Astronomy, Vanderbilt University, VU Station B 1807, Nashville, TN 37235, USA

${ }^{2}$ Fisk-Vanderbilt Masters-to-PhD Bridge Program Graduate Fellow; eugenio.v.garcia@gmail.com

${ }^{3}$ Harvard-Smithsonian Center for Astrophysics; 60 Garden Street, Mail Stop 20, Cambridge, MA 02138, USA
} 
where $R_{p}$ is the radius of the more massive primary star and $R_{s}$ is the radius of the less massive secondary star. These radii differ from the previously reported values of $R_{p}=5.23 \pm 0.06$ and $R_{s}=4.32 \pm 0.07$ by Hensberge et al. (2000). While these differences are not highly statistically significant given the uncertainties quoted in the two studies, the difference is slighly more than $1 \sigma$ and any difference is unexpected since Torres et al. (2010) recalculated the radii from the same data originally presented by Hensberge et al. (2000).

As is standard for the determination of EB radii, the radii are calculated from the volumetric radius which is defined as $R=\left(\frac{3}{4 \pi} V\right)^{1 / 3}$, where $V$ is the volume of the star. Therefore we suspected the difference may lie in different approaches to calculating the stellar volumes, which are themselves derived from the stellar potential surfaces. Since Torres et al. (2010) adopted the same stellar potentials originally reported by Hensberge et al. (2000), the different radii reported by the two authors is likely to be in the formulation used to transform the potentials to volumes.

Torres et al. (2010) computes $V$ using the stellar potentials, $\Omega$, and the stellar mass ratio, $q \equiv M_{2} / M_{1}$, from Hensberge et al. (2000) assuming a circular synchronous orbit. More formally, $V=V(\Omega, q)$ if the orbit is circular $(e=0)$, and if the stars rotate synchronously with the orbit $\left(F_{1}=F_{2}=1.0\right.$, where $F$ is the ratio of the stellar angular velocity to the orbital angular velocity). Following Kopal (1989):

$$
\Omega=\frac{1}{r}+q\left\{\frac{1}{\sqrt{1-2 \lambda r+r^{2}}}-r \lambda\right\}+\frac{q+1}{2} r^{2}\left(1-\nu^{2}\right)
$$

where $\nu=\cos \theta, \lambda=\cos \phi \sin \theta, \theta$ and $\phi$ are instantaneous direction angles between the two stars, and $r$ is the distance to the surface of the star from the star's center in the $(\theta, \phi)$ direction and in units of the instantaneous separation between the two stars. Through several complex mathematical operations, equation 1 can be used to uniquely define $V=V(\Omega, q)$ and therefore $R=R(\Omega, q)$ (see Equation 2.18 in Kopal 1989).

However, V578 Mon has an eccentric (i.e., non-circular) orbit with pseudo-synchronous rotation, where $e=0.0867, F_{1}=1.13$ and $F_{2}=1.11$ (Hensberge et al. 2000). Therefore the $R$ cannot be uniquely determined as a function of $\Omega$ and $q$ alone. Instead of using equation 1 , it is necessary to use $\Omega$ defined for non-circular orbits (Wilson 1979):

$$
\Omega=\frac{1}{r}+q\left\{\frac{1}{\sqrt{\delta^{2}-2 r \lambda \delta+r^{2}}}-\frac{r \lambda}{\delta^{2}}\right\}+F^{2} \frac{q+1}{2} r^{2}\left(1-\nu^{2}\right)
$$

where $\delta=D / a$ is the instantaneous separation between the two stars normalized to the semi-major axis. Equation 2 thus defines $V=V(\Omega, q, F, \delta)$ and therefore $R=R(\Omega, q, F, \delta)$. Whereas Torres et al. (2010) calculated the V578 Mon radii via equation 1, Hensberge et al. (2000) uses the Wilson-Devinney code Wilson \& Devinney 1971; Wilson 1979) which correctly incorporates equation 2 in computing the radii.

As an independent check on the Wilson-Devinney based calculation of the radii, we compute $R_{p}$ and $R_{s}$ by solving equation 2 numerically for $r(\theta, \phi)$ in unit steps of $\theta$ and $\phi$. We set $\Omega_{1}, \Omega_{2}, F_{1}, F_{2}, q$ and $e$ to values from Hensberge et al. (2000). For noncircular orbits, $\Omega=\Omega(t)$ since the instantaneous separation between the two stars varies over the orbit. We compute the volumetric radius at periastron, setting the instantaneous separation $\delta=1-e$, since the $\Omega$ reported by Hensberge et al. (2000) are the potentials at periastron (Wilson 1979). We compute $V$ from $r(\theta, \phi)$ discretely as:

$$
V=\frac{1}{3} \sum \sum r(\theta, \phi)^{3} \sin (\theta) \Delta \phi \Delta \theta
$$

where $\Delta \phi$ and $\Delta \theta$ are discrete steps in $\phi$ and $\theta$.

From equation 3 we compute $R_{p}=5.24$ and $R_{s}=4.33$, which are essentially identical to the radii from Hensberge et al. (2000). Finally, in the same fashion we also solve for $r(\theta, \phi)$ for the case of a circular orbit 
(equation 1), and compute $V$ using equation 3. In this case, we find $R_{p}=5.15$ and $R_{s}=4.21$, which are essentially identical to the radii reported in Torres et al. (2010).

Thus, it is clear that the differences in the radii reported for V578 Mon between Hensberge et al. (2000) and Torres et al. (2010) arises from an incorrect use by Torres et al. (2010) of the formulation for the stellar volumes assuming circular, synchronous orbits.

\section{Summary}

We have shown that the radii reported for V578 Mon by Torres et al. (2010) are incorrect, with an error of $\sim 2 \%$ relative to the expected values (Hensberge et al. 2000). While small, the error is unexpected since Torres et al. (2010) used the same data as Hensberge et al. (2000), and moreover the highest quality radius determinations of EBs should achieve $\sim 1 \%$ precision. We have traced the error to the use by those authors of the formulation for the stellar potential surfaces that is correct only in the case of circular, synchronous orbits. However, V578 Mon possesses an eccentric, pseudo-synchronous orbit, and thus its volumetric radii must be calculated from the formulation for the stellar potentials that correctly accounts for this (Wilson 1979).

Radii for V578 Mon were originally reported by Hensberge et al. (2000), who use the Wilson-Devinney code which incorporates the correct formulation for the stellar potentials in an eccentric orbit (equation 2). We have performed an independent check on the Hensberge et al. (2000) radii by solving equation 2 numerically; we recover radii identical to Hensberge et al. (2000). We are able to recover the Torres et al. (2010) radii when we solve the equations assuming a circular, synchronous orbit.

We have checked that the use of the erroneous formulation for the potential in Torres et al. (2010) does not significantly alter the radii for any other systems reported in that paper; fortunately only V578 Mon is sufficiently massive and eccentric to have been affected. A forthcoming paper (Garcia et al., in prep.) will report improved radii of V578 Mon taking into account an updated eccentricity of $e=0.07755$ and apsidal

motion $\dot{\omega}=0.07089 \mathrm{deg}$ cycle $^{-1}$ from Garcia et al. (2011). In the meantime we recommend the use of the radii from Hensberge et al. (2000) which we have corroborated here.

\section{REFERENCES}

Garcia, E. V., Stassun, K. G., Hebb, L., Gómez Maqueo Chew, Y., \& Heiser, A. 2011, AJ, 142, 27

Hensberge, H., Pavlovski, K., \& Verschueren, W. 2000, A\&A, 358, 553

Kopal, Z. 1989, Astrophysics and Space Science Library, 152

Pavlovski, K., \& Hensberge, H. 2005, A\&A, 439, 309

Torres, G., Andersen, J., \& Giménez, A. 2010, A\&A Rev., 18, 67

Wilson, R. E. \& Devinney, E. J. 1971, ApJ, 166, 605

Wilson, R. E. 1979, ApJ, 234, 1054 
Table 1. V578 Mon Radii Comparisons

\begin{tabular}{lrr}
\hline \hline & & \\
\hline & $R_{p}$ & \multicolumn{1}{c}{$R_{s}$} \\
\hline Hensberge et al. (2000) & $5.23 \pm 0.06$ & $4.32 \pm 0.07$ \\
\hline Torres et al. (2010) & $5.149 \pm 0.091$ & $4.21 \pm 0.10$ \\
\hline This Study & 5.24 & 4.33 \\
\hline & & \\
\hline \hline
\end{tabular}

